\title{
Distance Geometry in Active Structures
}

\author{
Josep M. Porta ${ }^{\dagger}$, Nicolás Rojas ${ }^{\ddagger}$, and Federico Thomas ${ }^{\dagger}$
}

\begin{abstract}
Distance constraints are an emerging formulation that offers intuitive geometrical interpretation of otherwise complex problems. It has applications in problems such as position and singularity analysis and path planning of mechanisms and structures. This paper reviews the recent advances in Distance Geometry providing a unified view of these apparently disparate problems. This survey reviews algebraic and numerical techniques and up to our knowledge, it is the first attempt to summarize the different approaches relaying in distance-based formulations.
\end{abstract}

\section{Introduction}

A structure can be seen as a complex multibody system. While rigid structures have been widely used in construction, passive mobile structures are commonly used, for instance, as shock absorbers. The advent of automation, though, opened the possibility to build active structures [29], i.e., structures which can actively vary their geometry as needed (see Fig. 1). Such structures are mechanisms since their motions are typically achieved by means of actuated elements like revolute joints of variable-length bars. Due to their shape versatility, variable geometry structures have a myriad of potential applications including robot arms [20], hyper-redundant manipulators [10], flight simulators [54], payload vibration reduction [14], the manipulation of large

\footnotetext{
† Institut de Robòtica i Informàtica Industrial, CSIC-UPC, Barcelona, Spain

$¥$ Department of Engineering and Design, University of Sussex, United Kingdom e-mail: \\{porta,fthomas \\}@iri.upc.edu,n.rojas@sussex.ac.uk Authors are in alphabetical order.
} 


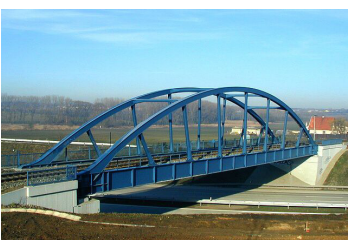

(a)

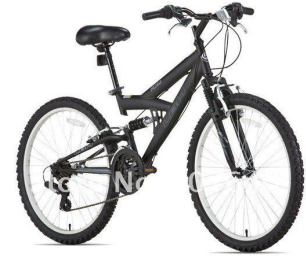

(b)

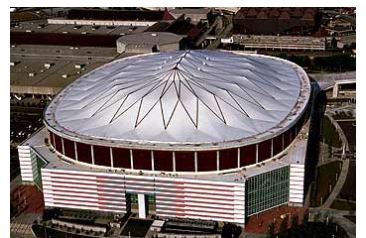

(c)

Fig. 1 Structures are typically composed by interconnected rigid bodies and they can have different types of mobility. (a) A fixed bridge is an example of rigid structure. (b) The shock absorbers of a bike are passive mobile structures (c) Retractable roofs are active structures.

payloads [52], morphing wings [16], space applications [30] or civil engineering structures [22].

The design of novel variable geometry structures rely on having a complete characterization of their valid configurations. Such configurations are defined by a system of equations encoding the assembly, task, or contact constraints intervening in the problem, and the goal is to analyze the motion capabilities by studying the solutions and properties of such system. The equations can be encoded with different formulations and the analysis can be significantly simplified if the right formulation is chosen.

The dominant formulation is based on homogeneous transforms using the parameters proposed by [15]. This formulation encodes the relative relation between the reference frames associated with the bodies connected by a given joint. While the motion simulation using such parameters is straightforward, the motion analysis using them is challenging because the resulting equations typically involve complex trigonometric expressions.

In the past few years, several works have shown that deviating from this classical approach and formalizing the motion analysis problems using distance constraints can be very advantageous. Distance constraints provide intuitive geometric insights on aspects of the motion analysis problem which are difficult to discern otherwise. Moreover, these geometric insights allow deriving solutions common to problems that otherwise have to be treated case-by-case $[33,47,46]$.

In some mechanisms, the configuration space is composed by isolated points. This is what happens, for example, when solving the position analysis problem of serial or parallel manipulators. In other situations, the valid configurations form a variety and the problem is to analyze relevant subsets of this variety. This is the case when analyzing the singularity loci. In systems in which the dimension of the variety is very high, the global analysis of the configuration space is unfeasible and the main problem is to find collision and/or singularity free paths connecting any two given configurations. 

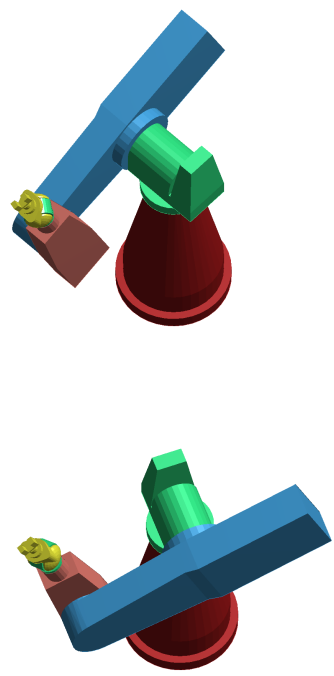
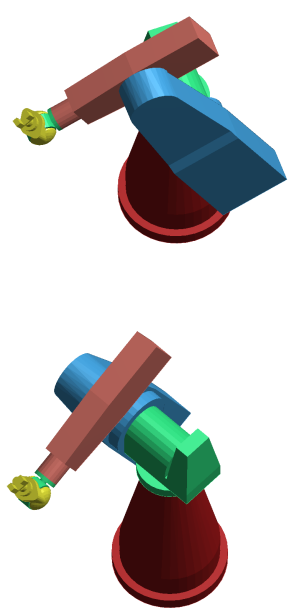

Fig. 2 The inverse kinematic problem of a serial robot consist in finding the robot configurations that position the end effector in a given pose. For the manipulator in the figure this problem has eight different solutions. Four of them are shown here.

Next, we review the existing Distance Geometry approaches to these three fundamental problems.

\section{Position Analysis}

The position analysis problem consists in computing all the valid configurations of a constrained multibody system. This problem appears for instance in the inverse kinematic of serial manipulators [27], as illustrated in Fig. 2. It appears also when solving the forward kinematics of parallel structures [28], when planning the motion of deployable structures [17], in robot grasping [49], in constraint-based object positioning [42], or in simultaneous localization and map-building [31]. The problem also appears in other domains, such as in the dynamical simulation of multi-body systems [21], in parametric computer-aided design (CAD) [3], or in the conformational analysis of biomolecules [60].

Traditional approaches translate the original geometric problem into a system of kinematic equations derived from the independent kinematic loops in the problem. Existing techniques to solve such systems of equations can be classified into algebraic or numerical methods. The use of independent loop equations has seldom been questioned despite the resulting expressions 

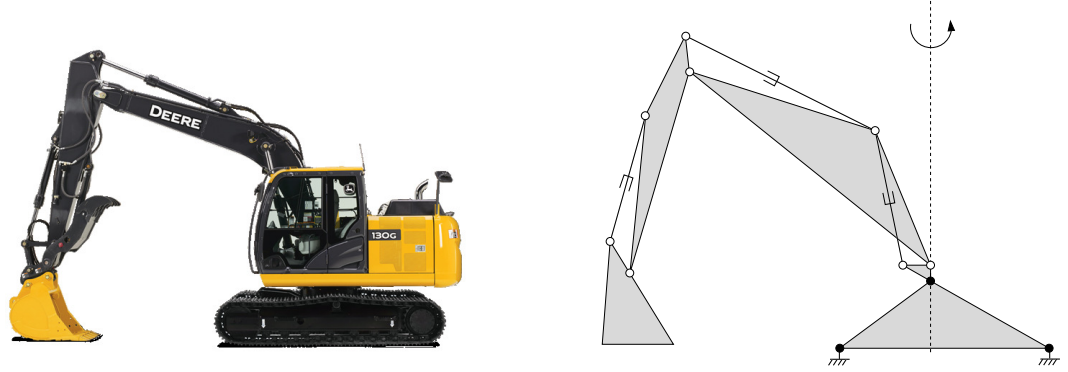

Fig. 3 An excavator (left) can be modeled as a planar mechanism (right) with a global rotation about a vertical axis. While and black dots represent movable and fixed revolute joints, respectively.

are not particularly suited neither for the algebraic nor for the numerical methods. First because arbitrary reference frames has to be included, and in second term because all formulas involve translations and rotations simultaneously thus leading to complex trigonometric equations. The distance-based formulation offers an alternative free from these two problems and has been shown to provide novel insights in both algebraic and numerical methods.

\subsection{Algebraic Methods}

Algebraic methods use variable elimination to reduce the initial system to a univariate polynomial whose roots, once backsubstituted into other equations, yield all solutions of the original system [12].

To apply the variable elimination methods to the equations resulting from the kinematic loops, the trigonometric expressions must be replaced to obtain a system of polynomial equations. Typically the tangent-half angle substitution is used, but it misses possible roots at $\pm \pi$. Moreover, the resulting expressions are complicated, limiting the scalability of the approach. Some of the successful results obtained with this approach can be attributed to clever manual manipulation of the expressions, which are difficult to generalize [40]. Using a distance based approach, the original geometric problem is translated into a graph where the nodes are points on the structure and the edges are distance, area, or volume constraints involving these points. Relying on this this formulation, Rojas and Thomas [43] proposed a procedure to solve the position analysis of complex planar mechanisms (see Fig. 3). The procedure analyzes the two possible assemblies of a triangle, given the distances between its vertices. This basic problem is elegantly formulated using the so-called bilateration matrices. Then, more complex mechanisms are analyzed decomposing them in strips of triangles and chaining bilateration 
matrices for triangles sharing one edge. This directly produces a scalar algebraic resultant in most cases, which can be transformed into a polynomial by clearing radicals.
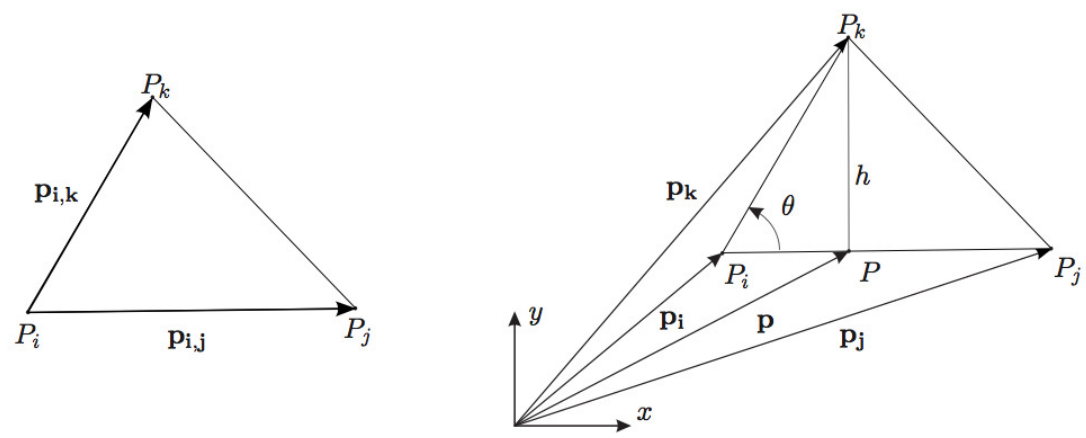

Fig. 4 The bilateration problem (left) and the associated notation (right).

Let us review this procedure in more detail. According to the notation in Fig. 4, the objective of the bilateration operation is to determine the location of point $P_{k}$ given known locations for points $P_{i}$ and $P_{j}$. The position vector of the orthogonal projection of $P_{k}$ onto $\overline{P_{i} P_{j}}$ can be expressed as

$$
\mathbf{p}=\mathbf{p}_{i} \sqrt{\frac{D(i, k)}{D(i, j)}} \cos \theta \mathbf{p}_{i, j}=\mathbf{p}_{i}+\frac{D(i, j ; i, k)}{D(i, j)} \mathbf{p}_{i, j}
$$

where

$$
D\left(i_{1}, \ldots, i_{n} ; j_{1}, \ldots, j_{n}\right)=\left|\begin{array}{cccc}
0 & 1 & \ldots & 1 \\
1 & s_{i_{1}, j_{1}} & \ldots & s_{i_{1}, j_{n}} \\
1 & \vdots & \ddots & \vdots \\
1 & s_{i_{n}, j_{1}} & \ldots & s_{i_{n}, j_{n}}
\end{array}\right|,
$$

with $s_{i, j}$ the squared of $d_{i, j}$, the distance between $P_{i}$ and $P_{j}$ is the CayleyMenger bideterminant of $n$ points and $D\left(i_{1}, \ldots, i_{n}\right)=D\left(i_{1}, \ldots, i_{n} ; i_{1}, \ldots, i_{n}\right)$ is the Cayley-Menger determinant.

Using $\mathbf{p}$, the position of $P_{k}$ can be expressed as

$$
\mathbf{p}_{k}=\mathbf{p} \pm \frac{\sqrt{D(i, j, k)}}{D(i, j)} \mathbf{S} \mathbf{p}_{i, j}
$$

where $\mathbf{S}=\left(\begin{array}{cc}0 & -1 \\ 1 & 0\end{array}\right)$ and the \pm sign accounts for the two mirror symmetric locations of $P_{k}$ with respect to the line defined by $\overline{P_{i} P_{j}}$. Substituting (1) in (3) we get 


$$
\mathbf{p}_{i, k}=\frac{D(i, j ; i, k)}{D(i, j)} \mathbf{p}_{i . j}+\frac{D(i, j, k)}{D(i, j)} \mathbf{S} \mathbf{p}_{i, j},
$$

which can be expressed in a more compact form as

$$
\mathbf{p}_{i, k}=\mathbf{Z}_{i, j, k} \mathbf{p}_{i, j}
$$

where

$$
\mathbf{Z}_{i, j, k}=\frac{1}{D(i, j)}\left(\begin{array}{cc}
D(i, j ; i, k) & \pm \sqrt{D(i, j, k)} \\
\pm \sqrt{D(i, j, k)} & D(i, j ; i, k) .
\end{array}\right)
$$

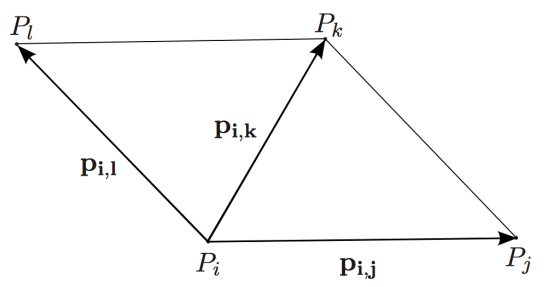

Fig. 5 Two triangles sharing one edge.

Note that (5) expresses $\mathbf{p}_{i, k}$ as a function of $\mathbf{p}_{i, j}$. When we have a strip of triangles, i.e. a sequence of triangles each sharing one edge with the previous one in the sequence, we can chain the bilateration process, i.e., multiply bilateration matrices, and express a vector in the final triangle as a function of a vector in the initial one. In the same way, we can derive expressions for vectors between points whose relative distance is initially unknown. For instance, in the situation in Fig. 5 we have that

$$
\mathbf{p}_{j, l}=\mathbf{p}_{i, l}-\mathbf{p}_{i, j}=\left(\mathbf{Z}_{j, k, l} \mathbf{Z}_{i, j, k}-\mathbf{I}\right) \mathbf{p}_{i, j} .
$$

Then, it can be shown that the squared distance between $P_{i}$ and $P_{l}$ is given by

$$
s_{j, l}=\operatorname{det}\left(\mathbf{Z}_{j, k, l} \mathbf{Z}_{i, j, k}-\mathbf{I}\right) s_{i, j},
$$

which expresses $s_{j, l}$ as a function of known edge lengths.

For larger problems the same procedure can be applied identifying strips of triangles with known edge lengths fully covering the mechanism. In most mechanisms with mobility zero, this process typically requires the introduction of unknown distances. Actually, the number of unknown distances to introduce coincides with the coupling number of the mechanism. Thus, the method can be applied to linkages with coupling number higher than one. In the final expression one of the distances in the triangle at the end of the strip is expressed as a function of an unknown distance in the first triangle. Thus, this expression is directly a scalar algebraic equation which can be converted 
into a polynomial by clearing radicals. This direct procedure to derive resultant polynomials is significantly simpler than the ones in the literature and it allows solving problems such as that in Fig. 6 which contains six independent kinematic loops, a number that has not been attained with elimination methods [61].
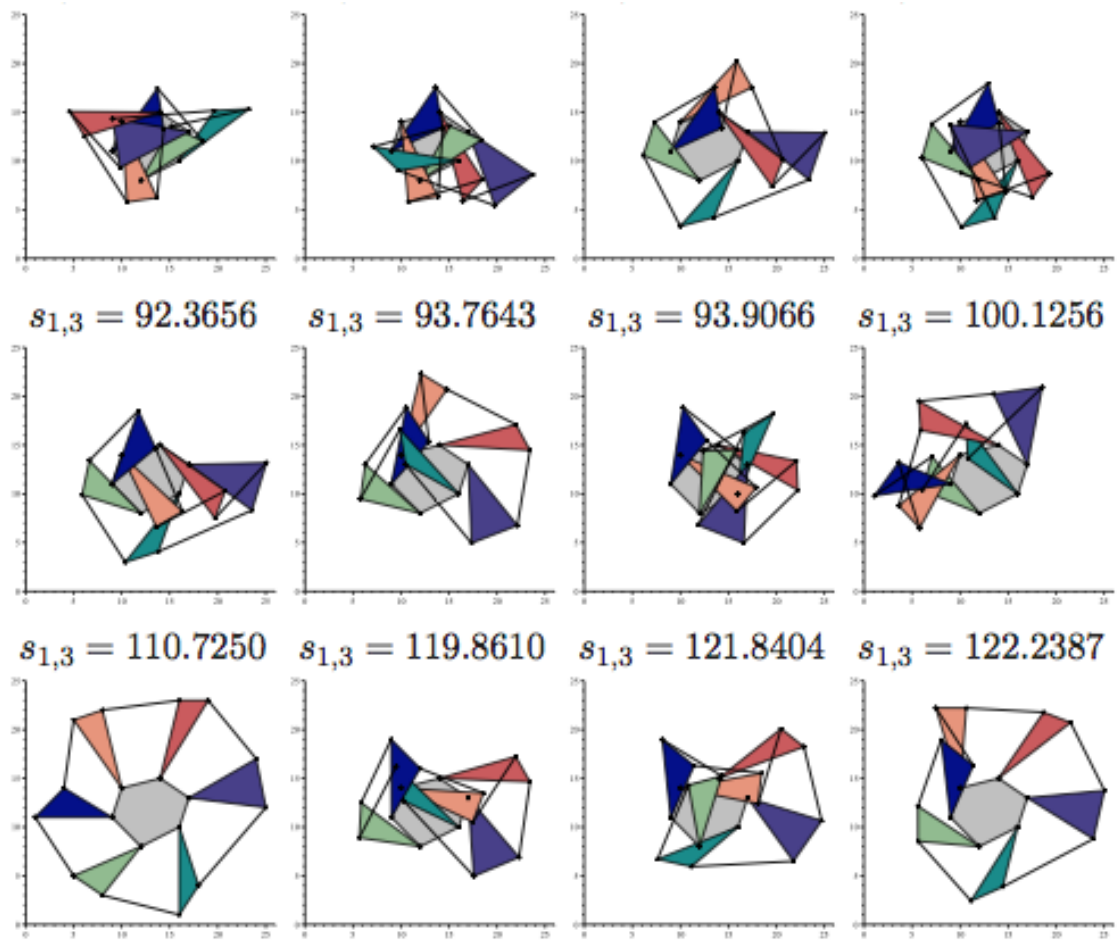

$$
s_{1,3}=130.0000
$$

$s_{1,3}=130.1666$

$s_{1,3}=134.9545$

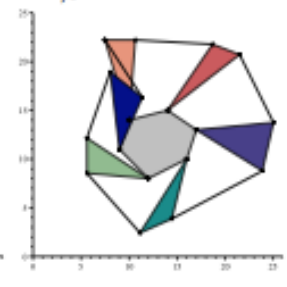

Fig. 6 Some of the valid configurations of a 13-link Watt-Baranov truss. The position analysis of this mechanism using algebraic methods has been shown to be feasible when formulating the problem in terms of distance constraints [43].

For systems with mobility 1, we obtain a polynomial depending on two unknown distances which can be used for path tracking: one of the unknowns is fixed and the system is solved for the remaining variable. The advantage of using a distance formulation in this case is that path crossing conditions can be readily identified since they correspond to alignments of particular points in the problem. Thus, the distance-based formulations significantly simplify the path tracking procedures, as compared with previous approaches [45].

This distance-based algebraic approach can be generalized to 3D using fans of tetrahedra instead of strips of triangles. This allows solving in closed 
form the position analysis of variable geometry trusses much more complex than those solvable with traditional formulations [38].

\subsection{Numerical Methods}

Although the distance-based algebraic methods have proved quite efficient in fairly non-trivial position analysis problems, this technique explodes in complexity with the size of the problem. Thus, to address larger problems one has to recourse to numerical techniques. Several distance-based alternatives are available in the literature.

\subsubsection{Trilateration methods}

The position analysis problem can be seen as that of determining all the possible values for the unknown distances in the graph of distance constraints encoding the problem. If the graph is represented in the form of an adjacency matrix, the problem boils down to complete the matrix from the distances initially fixed. Once the matrix is completed, standard linear algebra methods can be used to give coordinates to the points in the problem [4].
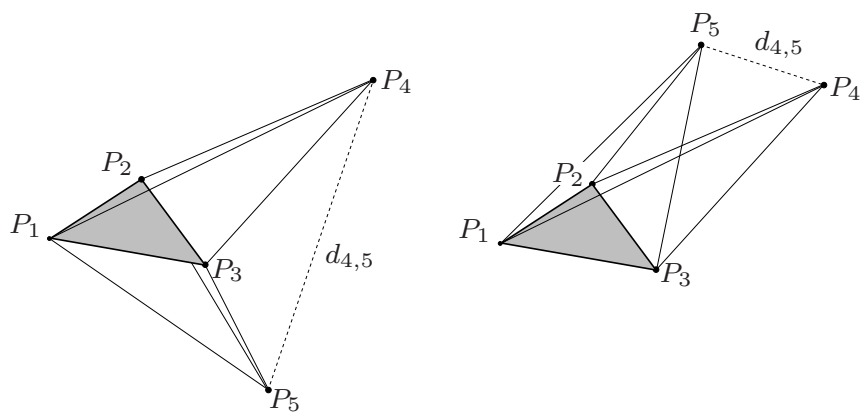

Fig. 7 Trilateration can be used to compute the distance between $P_{4}$ and $P_{5}$ from their distances to $P_{1}, P_{2}$, and $P_{3}$, which form a fixed triangle. Two solutions are possible depending on the location of $P_{4}$ and $P_{5}$ with respect to the plane defined from points $P_{1}$, $P_{2}$, and $P_{3}$

In some problems, the matrix completion process can be done in an incremental constructive way. In $\mathbb{E}^{3}$, if the relative distances between five points are all known except one, this unknown distance can be readily determined by trilateration. Using the notation in Fig. 7, we have that 
$s_{4,5}=\frac{2}{D(1,2,3)}\left(\left.D(1,2,3,4 ; 1,2,3,5)\right|_{s_{4,5}=0} \pm \sqrt{D(1,2,3,4) D(1,2,3,5)}\right)$.

Observe that no point coordinates appear in the result, only inter-point distances, and that two solutions are possible, corresponding to the two possible signs for the square root in the expression.

A problem is trilaterable if it is possible to determine a trilateration sequence to compute the initially unknown distances in the problem. This sequence can be readily determined by subgraph matching, i.e., finding parts of the original graph which match with the trilateration subgraph shown in Fig. 7. Porta et al. [33] showed that the inverse/direct kinematics of the most usual serial/parallel robots are trilaterable problems, which greatly simplifies their resolution. Moreover, [32] shows that if the searched subgraph includes six points with only one unknown distance between them, this unknown distance is linear with respect to the rest of distances and, thus, it has only one possible solution. This avoids the generation of distance completions that have to be discarded in a post-processing stage since they would include tetrahedra with orientations incompatible with the original problem. For more details on the role of orientations in Distance Geometry see Section 2.2.3.

Note that the trilateration process is closely related with the algebraic approach described in Section 2.1. The main difference is that in the trilateration only one new unknown distance appears at each step while in the algebraic method, the first step involves two unknown distances. This is why the former method is purely numerical while the latter generates an symbolic expression in one variable distance.

To the best of our knowledge, the combination of the trilateration step and the procedure to determine a trilateration sequence were first introduced by [33, 32] and, latter on, independently proposed by [25] in the context of Structural Biology, but in Cartesian space, i.e., relying on the coordinates of the points and assigning coordinates to the trilaterated point at each step. More recently, [26] proposed an equivalent algorithm in distance space.

\subsubsection{Branch-and-Prune methods}

Unfortunately, not all problems admit a trilateration sequence and, thus, general solvers to determine the valid distance matrices from the initially known distances must be devised. Porta et al. present alternative general solvers relying on a branch-and-prune technique [35, 36, 37]. These approaches iteratively eliminate regions of the space of distances where the distance constraints are not satisfied. When the distance space can not be further reduced, it is split, and the reduction and split procedure is recursively applied to the two resulting sub-spaces. This process can be seen as an extension of the classic bound-smoothing techniques [13] and isolates the valid solutions for the in- 
put problem in the form of interval matrices at the desired resolution. Since splitting the search space is trivial, the key operation of the branch-and-prune methods is the procedure used to shrink the boxes in distance space.

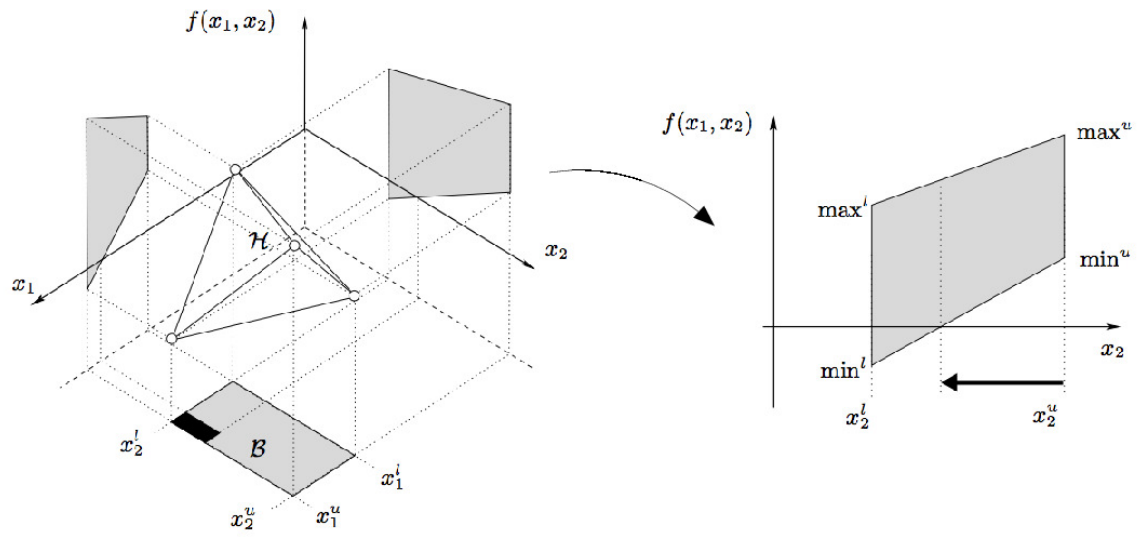

Fig. 8 Segment-trapezoid clipping. Left: In this two-variable case, the graph of the multilinear function $f\left(x_{1}, x_{2}\right)$ in the domain box $\mathcal{B}$ necessarily lies inside the shown tetrahedron $\mathcal{H}$. The vertices of $\mathcal{H}$ are obtained by evaluating $f$ in the corners of $\mathcal{B}$. The projection of $\mathcal{H}$ to a given coordinate planes defines a trapezoid. Right: From the initial range for a variable, we can prune all points for which its trapezoid does not intersect the $f\left(x_{1}, x_{2}\right)=0$ line.

The method presented in $[36,37]$ introduces variable substitutions to convert the quadratic expression resulting from the Cayley-Menger determinants into multilinear equations. The graph of a multilinear function defined on an axis-aligned box is included in the convex hull of the evaluation of the function in the corners of the domain [41]. Then, the solution of $f(\mathbf{x})=0$ can be bounded to the intersection of this hull with the plane $f(\mathbf{x})=0$. Since the computation of this intersection can be difficult, the method projects the hull onto each coordinate plane, as depicted in Fig. 8(left), and intersect each of the resulting trapezoids with the line, as shown in Fig. 8(right). Usually, these segment-trapezoid clippings reduce the ranges of some variables giving a smaller box (the black rectangle in Fig. 8) that still bounds the root locations. Although this strategy produces less pruning than the convex hullplane clipping, in practice it results advantageous due to the lower cost of its operations.

A Gough-Stewart platform is a 6 degrees of freedom structure composed of a moving platform connected to a base by six legs. The pose of the platform is controlled by the leg lengths. Parallel structures are used in many applications including positioning tools [58], flight simulators [9], or radiotelescopes [53]. After some leg rearrangements (see Section 3), the forward kinematic problem 

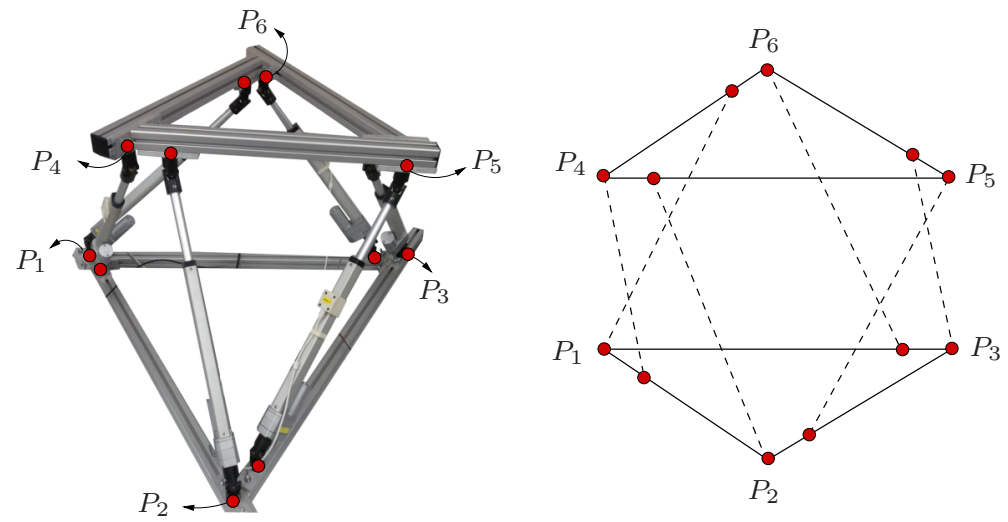

Fig. 9 Left: A hexapod structure. Right: Associated distance graph where nodes are points and edges are distance constraints. Solid lines denote distances that are constant independently of the configuration.

of the Gough-Stewart in Fig. 9 can be formulated using two Cayley-Menger determinants [44]

$$
\begin{aligned}
& D(1,3,4,5,6)=0 \\
& D(1,2,4,5,6)=0
\end{aligned}
$$

where $P_{1}, P_{2}$, and $P_{3}$ are the points defining the triangle at the base of the structure and $P_{4}, P_{5}$, and $P_{6}$ the vertices in the triangle at the moving platform. The branch-and-prune algorithm proposed by [37] determines the two solutions of this problem typically in less than 0.01 seconds on a standard desktop computer.

A variation of the above algorithm where the box reduction is based on the properties of the Bernstein polynomials has been used to elucidate the valid conformations of several molecular structures [34]. This method was parallelized and run on the MareNostrum supercomputer ${ }^{1}$ to obtain the first complete description of the conformational space of the cyclooctane, which is a two-dimensional variety. To the extent of our knowledge, this was the first Distance Geometry method able to characterize such complex solution spaces.

Distance Geometry provides yet another alternative to crop the distance ranges based on the reduction and expansion of the dimension of the problem [56]. This approach is purely geometric, avoiding the algebraization of the problem. Taking the vector from $P_{1}$ to $P_{n}$ as a reference, a vector $\mathbf{q}=\left(\bar{d}_{1, n}, \ldots, \bar{d}_{n, n}\right)$ can be defined where

\footnotetext{
${ }^{1}$ http://www.bsc.es/marenostrum-support-services
} 


$$
\bar{d}_{i, n}=\frac{1}{2 d_{1, n}}\left(d_{i, n}^{2}+d_{1, n}^{2}-d_{i, 1}^{2}\right)
$$

is the orthogonal projection of vector from $P_{n}$ to $P_{i}$ onto the reference vector. We can also define the orthogonal complement of this projection which is a matrix $\mathbf{Q}^{\perp}$ with

$$
\mathbf{Q}_{i, j}^{\perp}=d_{i, j}^{2}-\frac{1}{4 d_{1, n}^{2}}\left(d_{i, n}^{2}-d_{j, n}^{2}+d_{j, 1}^{2}-d_{i, 1}^{2}\right)^{2} .
$$

It can be shown that $\mathbf{Q}$ is a proper Euclidean distance matrix in $\mathbb{R}^{d}$ if and only if the $\mathbf{Q}_{i, j}^{\perp}$ with $i, j=1 \ldots n-1$ is a correct Euclidean distance matrix in $\mathbb{R}^{d-1}$. Thus, the method projects the input distance matrix with interval ranges until the problem becomes one-dimensional and, hence, consistency can be enforced using the triangular equality. The eventually reduced ranges is backprojected using the intermediate vectors $\mathbf{q}$ and matrices $\mathbf{Q}^{\perp}$ to find tighter ranges for the distances in the original problem. The direct evaluation of (10) and (11) using interval arithmetics would be inaccurate due to the well-known overestimations effect of this approach [1]. However, if a function $z=g(\mathbf{x})$ is monotone in an axis-aligned domain $\mathbf{x}=\left(x_{1}, \ldots, x_{n}\right)$, with $x_{i} \in$ $\left[x_{i}^{l}, x_{i}^{u}\right]$, and the derivatives of $g$ are available, its upper bound is $z^{u}=g(\hat{\mathbf{x}})$ where $\hat{\mathbf{x}}=\left(\hat{x}_{1}, \ldots, \hat{x}_{n}\right)$ is the vertex of the domain given by

$$
\hat{x}_{i}= \begin{cases}x_{i}^{l} & \text { if } \partial g / \partial x_{i}<0 \\ x_{i}^{u} & \text { otherwise }\end{cases}
$$

The lower bound, $z^{l}$, is in a vertex defined with the opposite criterion. Thus, tight bounds for $\bar{d}_{i, n}$ and $\mathbf{Q}_{i, j}^{\perp}$ can be obtained analyzing their respective derivatives. This method has been applied to solve the position analysis of planar and spatial mechanisms with mobility 0 and $1[56,39]$.

\subsubsection{Bound Smoothing with Orientation Constraints}

One of the main shortcomings of the approaches described in the previous section is its limited capability to encode orientation constraints. Let us consider the regional part of the wrist-partitioned 6R robot shown in Fig. 10(left), that is, the first three links and joints that permit locating the wrist center anywhere in the robot's workspace. Fig. 10(right) shows the formalization of this problem as a graph of distance constraints. In this example, any standard distance constraint solver would obtain, in general, eight different sets of compatible distances. Nevertheless, it is well-known that the inverse kinematics of a $3 \mathrm{R}$ robot can only have up to four solutions [57]. This apparent contradiction has a simple explanation: A distance-based technique would not take into account the relative orientations of the tetrahedra defined by 

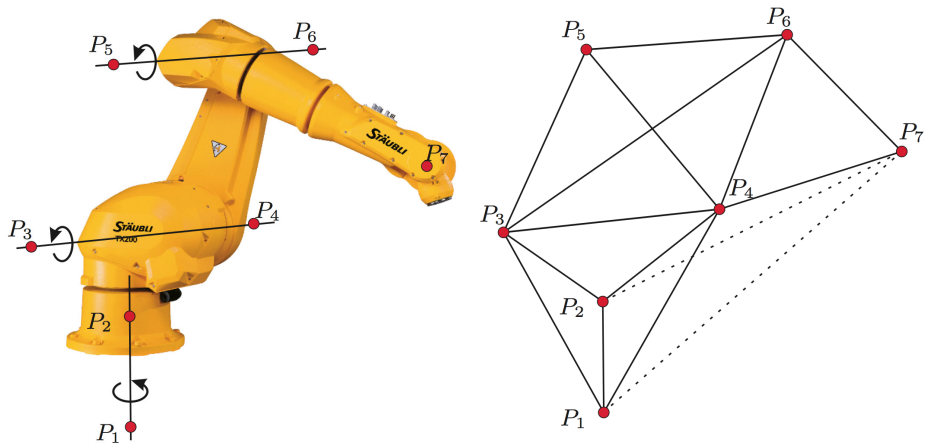

Fig. 10 Left: The configuration of the regional part of a wrist-partitioned $6 \mathrm{R}$ robot is determined by the location of seven points. Right: Associated distance graph where nodes stand for points and edges for known distances between the corresponding points. Solid lines represent constant distances, regardless of the location of the end-effector.

the sets of points $\left\{P_{1}, P_{2}, P_{3}, P_{4}\right\}$ and $\left\{P_{3}, P_{4}, P_{5}, P_{6}\right\}$. The same situation occurs in many other structures.

To address this issue, Rull et al. presents a distance bound smoothing approach that permits incorporating orientation constraints in the process of reducing the valid ranges of distances [50]. This approach focus on planar problems that are formalized with the following constraints:

- For all sets of three points:

$$
D\left(i_{1}, i_{2}, i_{3}\right) \leq 0
$$

- For all sets of four points:

$$
D\left(i_{1}, i_{2}, i_{3}, i_{4}\right)=0
$$

and

$$
D\left(i_{1}, i_{2}, i_{3} ; i_{1}, i_{2}, i_{4}\right)=\left\{\begin{array}{l}
<0 \text { if } \sigma_{i_{1}, i_{2}, i_{3}} \sigma_{i_{1}, i_{2}, i_{4}}>0 \\
\geq 0, \text { otherwise }
\end{array}\right.
$$

where $\sigma_{i, j, k}$ is defined as negative if points $P_{i}, P_{j}$, and $P_{k}$ have to be arranged clockwise and positive otherwise.

While the expansion of (12) leads to the triangular inequality involving the distances between $P_{i_{1}}, P_{i_{2}}$, and $P_{i_{3}},(13)$ is nothing else than the tetrangular equality involving the six pairwise distances between $P_{i_{1}}, P_{i_{2}}, P_{i_{3}}$, and $P_{i_{4}}$.

Note that the whole set of orientation constraints in (14) can not be fixed arbitrarily. Actually, it is possible to define a basis that determines all other orientations [55].

Since an efficient algorithm exists for tightening bounds using triangular constraints [19], it can be safely assumed that they are already satisfied by 


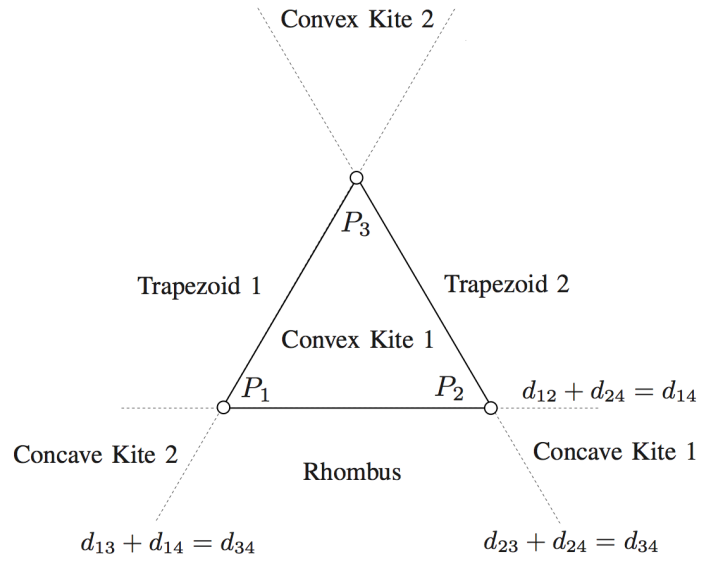

Fig. 11 Taking triangle $P_{1}, P_{2}$, and $P_{3}$ as a reference, the plane is divided in seven regions. If $P_{4}$ is bound to be in one of these regions, $s_{3,4}$ is monotone with respect to the rest of squared distances. The boundaries separating the monotonic regions correspond to configurations where there is an alignment of three points.

the initial ranges. Thus, the approach focuses on the analysis of (13) under orientation constraints. The expansion of this equality yields a quadratic expression in any of the involved squared distances. For instance, for the set of points $\left\{P_{1}, P_{2}, P_{3}, P_{4}\right\}$ we have

$$
s_{3,4}=\frac{\left.D(1,2,4 ; 1,2,3)\right|_{s_{3,4}=0}+\sigma_{1,2,4} \sigma_{1,2,3} \sqrt{D(1,2,4) D(1,2,3)}}{D(1,2)}
$$

or alternatively

$$
s_{3,4}=\frac{\left.D(1,2,4 ; 1,2,3)\right|_{s_{3,4}=0}+16 A_{3} A_{4}}{D(1,2)},
$$

where $A_{i}$ denotes the oriented area of the triangle defined by the ordered set $\left\{P_{1}, P_{2}, P_{3}, P_{4}\right\} \backslash P_{i}$, since $4 A_{i}=\sigma_{j, k, l} \sqrt{-D(j, k, l)}$.

The range of $s_{3,4}$ can be tightly bounded using the monotonicity analysis presented at the end of Section 2.2.2. To apply this method to the function in (16), we need to compute the derivatives of $s_{3,4}$ with respect to $s_{i, j}$. Instead of computing these derivatives form (16) it is more convenient to obtain them from the linearization of (13), which reads as:

$$
\begin{aligned}
& A_{1} A_{2} \delta s_{1,2}-A_{1} A_{3} \delta s_{1,3}+A_{1} A_{4} \delta s_{1,4} \\
+ & A_{2} A_{3} \delta s_{2,3}-A_{2} A_{4} \delta s_{2,4}+A_{3} A_{4} \delta s_{3,4}=0 .
\end{aligned}
$$

Then, we have that 

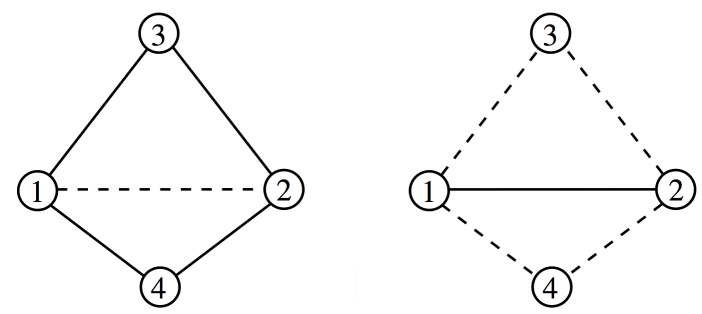

Fig. 12 The two configurations giving the lower (left) and upper (right) bounds for $s_{3,4}$ in the Rhombus region. Solid and dashed lines indicate distances at their lower and upper limits, respectively.

$$
\frac{\partial s_{3,4}}{\partial s_{i, j}}=-1^{i+j} \frac{A_{i} A_{j}}{A_{3} A_{4}} .
$$

As long as the sign of the oriented areas of the triangles defined by $P_{1}, \ldots, P_{4}$ do not change, $s_{34}$ is monotone. Therefore, in this case, we can readily identify the vertices providing tight bounds for $s_{3,4}$ by controlling the regions where there are orientation sign changes.

Fig. 11 shows a partition of the plane in regions where the orientations of the triangles defined by $P_{1}, P_{2}, P_{i}$ with $i>3$ are constant, taking the triangle defined by $P_{1}, P_{2}$ and $P_{3}$ as a reference. If $P_{4}$ remains in one of these regions, the bounds for $s_{3,4}$ can be readily determined. For instance, if $P_{i}$ is in the Rhombus region, the patterns in Fig. 12 identify the vertices of the domain giving a tight range for $s_{3,4}$. We can identify 14 patterns, 2 for each region, which subsume the 7 patterns used by [13].

When three points can be aligned within the allowed distance ranges the boundaries separating the monotonic areas must be recursively analyzed. At the end of the process, tight bounds for the variable of interest are obtained.

As an alternative, the geometric approach based on projections and backprojections introduced in [39] can also be extended to take into account orientation constraints. Moreover, this method can operate in 3D problems whereas the extension of the method introduced in [50] to 3D is not trivial.

The integration of the orientation constraints opens a new range of applications of Distance Geometry methods such as the coordination of robot teams, sensor data fusion, and constraint-based robot programming, to name just a few. For instance, Fig. 13 illustrates the application of this method to the mutual localization of a robot team.

\section{Singularity Analysis}

The singularity locus of a given mechanism are the configurations where mobility or control issues might arise. To prevent malfunctions or even structural 


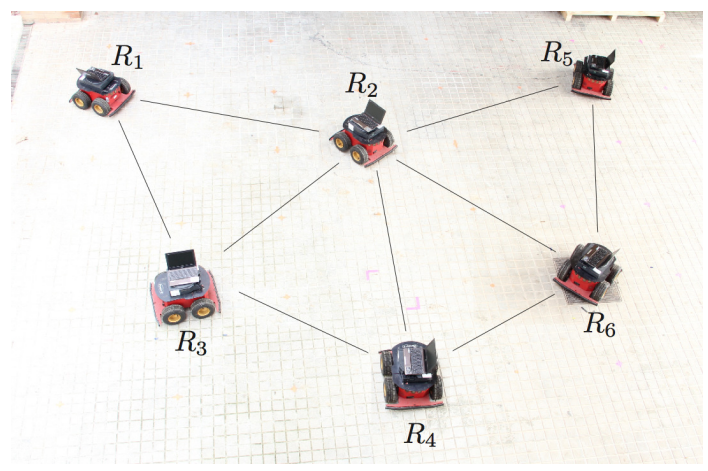

Fig. 13 A robot formation. Each robot is equipped with an ultrasound sensor to measure the distances to nearby teammates. The lines in the figure represent the distances actually measured. The orientation of the triangles is given by cameras mounted on the robots. The integration of the distance and orientation constraints permits determining tight bounds for the possible location of each robot.

damages singularities must be avoided. Thus, due to its relevance, singularity analysis is one of the main topics of research in mechanics. However, the characterization of singularities has only been achieved for particular mechanism instances or require the use of complex computational methods [6]. In general, modifications of the mechanism parameters change the singularity locus in unpredictable ways, which hinders the analysis of new structures. However, Borras and Thomas proposed Distance Geometry tools to identify singularity-invariant leg rearrangements for parallel structures, i.e., changes in the attachments of the legs to the base or the platform that do not change the singularity locus [7]. This generalizes the singularity analysis of a particular structure to all the other structures that can be defined from it with singularity-invariant leg rearrangements. Moreover, these rearrangements can be used to avoid multiple spherical joints, significantly simplifying the the actual construction of the structure [44].

In a parallel structure, the linear and angular velocity of the moving platform, $\mathbf{v}$ and $\boldsymbol{\Omega}$ respectively, are related with the leg lengths by

$$
\mathbf{R}_{l}\left(\begin{array}{c}
i_{1} \\
\vdots \\
i_{6}
\end{array}\right)=\mathbf{J}\left(\begin{array}{c}
\mathbf{v} \\
\mathbf{\Omega}
\end{array}\right)
$$

where $\mathbf{R}_{l}$ is a diagonal matrix with the leg lengths, $l_{1}, \ldots, l_{6}$, and $\mathbf{J}$ is the matrix of non-normalized Plücker coordinates of the six leg lines. The relevant singularities for parallel structure occur when $\operatorname{det}(\mathbf{J})=0$. Now assume that we rearrange the leg attachments and that the square of the new leg lengths, $d_{1}, \ldots, d_{6}$, are related to the previous ones by an affine relation of the form 


$$
\left(\begin{array}{c}
d_{1}^{2} \\
\vdots \\
d_{6}^{2}
\end{array}\right)=\mathbf{A}\left(\begin{array}{c}
l_{1}^{2} \\
\vdots \\
l_{6}^{2}
\end{array}\right)+\mathbf{b}
$$

then, as shown by [8], the relation between the change in the leg lengths and the velocity of the platform becomes

$$
\mathbf{R}_{d}\left(\begin{array}{c}
\dot{d}_{1} \\
\vdots \\
\dot{d}_{6}
\end{array}\right)=\mathbf{A} \mathbf{J}\left(\begin{array}{c}
v \\
\Omega
\end{array}\right)
$$

and the new singularity condition is $\operatorname{det}(\mathbf{A} \mathbf{J})=\operatorname{det}(\mathbf{A}) \operatorname{det}(\mathbf{J})$. If $\operatorname{det}(\mathbf{A})$ is a constant non-null factor, the leg rearrangement has no effect on the singularity locus. If $\operatorname{det}(\mathbf{A})$ is null, then the rearrangement introduces an architectural singularity, i.e., the resulting platform is in a singularity irrespectively of its leg lengths.
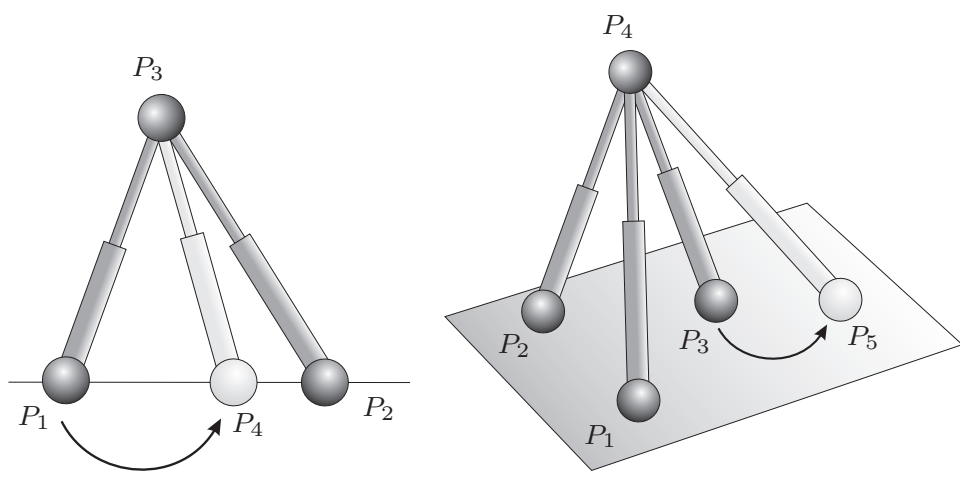

Fig. 14 Singularity invariant leg rearrangements. Left: Rearrangement along a line connecting two anchor points. Right: Rearrangement in a plane defined by three anchor points.

The Cayley-Menger determinants can be used to derive the affine relation between the leg lengths before and after the rearrangement. For instance, consider the situation in Fig. 14(left) where the anchor point $P_{1}$ is displaced along the line supported by $P_{1}$ and $P_{2}$. Since in this rearrangement the four points remain coplanar, then

$$
D(1,2,3,4)=\left|\begin{array}{lllll}
0 & 1 & 1 & 1 & 1 \\
1 & 0 & \left(d_{1,4}+d_{2,4}\right)^{2} & s_{1,3} & s_{1,4} \\
1 & \left(d_{1,4}+d_{2,4}\right)^{2} & 0 & s_{2,3} & s_{2,4} \\
1 & s_{1,3} & s_{2,3} & 0 & s_{3,4} \\
1 & s_{1,4} & s_{2,4} & s_{3,4} & 0
\end{array}\right|=0 .
$$


Expanding this determinant we obtain

$$
d_{2,4} s_{1,3}+d_{1,4} s_{2,3}-\left(d_{1,4}+d_{2,4}\right) s_{3,4}-d_{1,4} d_{2,4}\left(d_{1,4}+d_{2,4}\right)=0
$$

which defines a affine relationship between the leg lengths before and after the rearrangement with

$$
\mathbf{A}=\left(\begin{array}{cccc}
\frac{d_{2,4}}{d_{1,4}+d_{2,4}} & \frac{d_{1,4}}{d_{1,4}+d_{2,4}} & \ldots & 0 \\
0 & 1 & \ldots & 0 \\
\vdots & \vdots & \ddots & \vdots \\
0 & 0 & \ldots & 1
\end{array}\right)
$$

Since $\operatorname{det}(\mathbf{A})=d_{2,4} /\left(d_{1,4}+d_{2,4}\right)$, the proposed change in the anchor point location is a singularity invariant leg rearrangement.

Fig. 14(right) shows another possible leg rearrangement where a leg attachment is moved on the plane defined by the anchor points of three legs. In this case the conditions to be held is

$$
D(1,2,3,4,5)=0
$$

which can be rewritten to

$$
\begin{aligned}
& D(1,2,3 ; 2,3,5) s_{1,4}-D(1,2,3 ; 1,3,5) s_{2,4} \\
& +D(1,2,3 ; 1,2,5) s_{3,4}-D(1,2,3) s_{4,5}+C=0
\end{aligned}
$$

where $C$ is a constant that does not depend on the distances involving $P_{4}$. This leads to a singularity factor

$$
\operatorname{det}(\mathbf{A})=\frac{D(1,2,3 ; 2,3,5)}{D(1,2,3)},
$$

which is independent of the structure configuration and, thus, defines a singularity invariant leg rearrangement.

Note that other leg rearrangements are possible. The advantage of using Distance Geometry in their derivation is that, in general, the singularity factors have a direct geometric interpretation.

\section{Path Planning}

For structures with high mobility, the comprehensive description of both their configuration spaces and their singularity loci is unfeasible. Fortunately, in these cases, the research efforts usually focus on path planning problems, i.e., problems consisting in determining how to move the structure from an 
initial to a goal configuration while avoiding collisions or singularities [11, 23], although this second aspect is less treated in the literature [5].

For tree-like structures, the configuration space is parametric, which greatly simplifies the problem. However, when kinematic loops appear in the problem (see Fig. 15) the configuration space becomes a null-measure manifold embedded in the ambient space formed by the joint variables [24]. Actually, kinematic loops appears in many relevant problems such as complex manipulation problems [51], parallel robot path generation [28], grasp planning [48], or surgery planning [2].

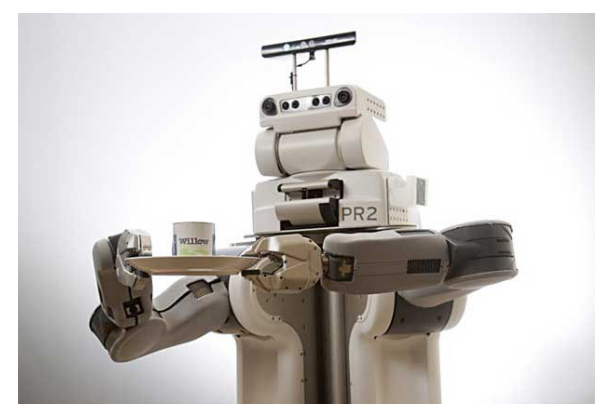

Fig. 15 A two-armed service robot holding a plate. The arms must be in contact with the plate and the plate must remain horizontal to avoid tilting the coffee mug on it. A loop of kinematic constraints is thus generated.

Under the presence of kinematic constraints, standard formulations produce involved configurations spaces. For some families of structures, though, distance constraints produce much simpler representations of the configuration spaces in which the path planning problem can be easily solved.

Trinkle and Milgram analyze the configuration space of planar chains with revolute joints formalized with joint angle parameters, but also using concepts from Distance Geometry [59]. Using the notion of long link, it proves that the configuration spaces of such mechanisms are connected if and only if they do not have three long links. Otherwise, their configuration space has two components and each of them is toroidal. Then, they propose a path planning algorithm differentiating between these two cases. When the configuration space has two components, the first task is to discern if the two configurations to connect are in the same component. If not, the path planning problem can not be solved. For configurations in the same component, one link is used to drive the mechanism while the remaining links comply in a series of accordion moves that can be proven to connect the configurations of interest. The final path is not optimal, but the algorithm is shown to be very efficient for significantly long loops.

Han et al. [18] proposes a distance-based formulation for planar closedchains with revolute joints, where a configuration is represented by the set 
of distances from a point on the base link to the end-points of the rest of links, complemented with a set of triangle orientations. In this parameter space the loop closure constraints become linear inequalities and the configuration space becomes practically piecewise convex. The boundaries between the different convex regions of this space are given by particular alignments of points, i.e., changes in the orientations of the triangles. Thus, to connect any two given configurations, one only needs to identify the boundaries to be crossed and define a piecewise linear path between them.

The approaches by Trinkle and Milgram [59] and by Han et al. [18] can be both generalized to spatial mechanism with spherical joints. However, no general joints nor joint limits can be encoded in these approaches, which hinders their general applicability.

\section{Summary}

Distance-based formulations provide new insights in fundamental problems. In the context of algebraic methods, the number of variable eliminations is reduced to the point in which, in many cases of practical interest, it is no longer required. Numerical methods also benefit from the use of distance formulations since trilateration approaches naturally follow from them and they provide a rich set of tools to reduce the search space in the context of branch-and-prune approaches. Moreover, distance formulations allow defining singularity invariant leg rearrangements, which significantly expand the utility of previous singularity characterizations in Gough-Stewart platforms. This allows the construction of active structures with well-characterized singularity loci and without complex mechanical pieces such as double spherical joints. Finally, in many cases, the analysis of the configuration spaces and the associated path planning problems can be certainly simplified when adopting a distance-based formulation.

Despite the extensive history of Distance Geometry is has only been recently introduced the position and motion analysis of structures. Given the success cases presented in this survey, we expect that the use of distance-based formulations and their associated tools will be soon generalized as a powerful alternative to address the complex geometric problems arising in this discipline. Finally, the Distance Geometry methods developed described in this survey are able to solve a wide variety of problems including trilaterable and non-trilaterable cases, linkages with coupling number higher than one, and even flexible graphs. We expect that these methods would find application in domains other than the ones presented here. 


\section{References}

1. Alefeld, G., Herzberger, J.: Introduction to Interval Computations. Academic Press, Orlando, Florida (1983)

2. Ballantyne, G., Moll, F.: The da Vinci telerobotic surgical system: Virtual operative field and telepresence surgery. Surgical Clinics of North America 83(6), 1293-1304 (2003)

3. Bettig, B., Hoffmann, C.M.: Geometric constraint solving in parametric computeraided design. ASME Journal of Computing and Information Science in Engineering 11, 021,001 (2011)

4. Blumenthal, L.M.: Theory and Applications of Distance Geometry. Oxford University Press (1953)

5. Bohigas, O., Henderson, M.E., Ros, L., Manubens, M., Porta, J.M.: Planning singularity-free paths on closed-chain manipulators. IEEE Transactions on Robotics 29(4), 888-898 (2013)

6. Bohigas, O., Zlatanov, D., Ros, L., Manubens, M., Porta, J.M.: A general method for the numerical computation of manipulator singularity sets. IEEE Transactions on Robotics 30(2), 340-351 (2015)

7. Borràs, J.: Singularity-invariant leg rearrangements on Stewart-Gough platforms. Ph.D. thesis, Institut de Robòtica i Informàtica Industrial (2011)

8. Borràs, J., Thomas, F., Torras, C.: Singularity-invariant leg rearrangements in doublyplanar Stewart-Gough platforms. In: Robotics Science and Systems (2010)

9. Cappel, K.L., Marlton, N.: Motion simulator (1967). U.S. patent 3295224

10. Chirikjian, G.S., Burdick, J.W.: A hyper-redundant manipulator. IEEE Robotics and Automation Magazine 1(4), 22-29 (1994)

11. Choset, H., Lynch, K., Hutchinson, S., Kantor, G., Burgard, W., Kavraki, L., Thrun, S.: Principles of Robot Motion: Theory, Algorithms, and Implementations. MIT Press (2005)

12. Cox, D., Little, J., O'Shea, D.: An Introduction to Computational Algebraic Geometry and Commutative Algebra, 2nd edition edn. Springer (1997)

13. Crippen, G., Havel, T.F.: Distance Geometry and Molecular Conformation. Research Studies Press (1998)

14. Dadone, P., Lacarbonara, W., Nayfeh, A.H., Vanlandingham, H.F.: Payload pendulation reduction using a variable-geometry-truss architecture with LQR and fuzzy controls. Journal of Vibration and Control 9(7), 805-837 (2003)

15. Denavit, J., Hartenberg, R.: A kinematic notation for lower-pair mechanisms based on matrices. Transactions of the ASME. Journal of Applied Mechanics 23, 215-221 (1955)

16. Finistauri, A.D., Fengfeng, X.: Type synthesis and kinematics of a modular variable geometry truss mechanism for aircraft wing morphing. In: International Conference on Reconfigurable Mechanisms and Robots, pp. 478-485 (2009)

17. Guest, S.: Deployable structures: Concepts and analysis. Ph.D. thesis, Cambridge University (1994)

18. Han, L., Rudolph, L., Blumenthal, J., Valodzin, I.: Stratified deformation space and path planning for a planar closed chain with revolute joints. In: S. Akella, N.M. Amato, W.H. Huang, B. Mishra (eds.) Algorithmic Foundation of Robotics VII, Springer Tracts in Advanced Robotics, vol. 47, pp. 235-250. Springer (2008)

19. Havel, T.: Distance geometry, pp. 1701-1710. Wiley, New York (1995)

20. Hughes, P.C., Sincarsin, W.C., Carroll, K.A.: Trussarm - A variable-geometry-truss manipulator. Journal of Intelligent Material Systems and Structures 2(2), 148-160 (1991)

21. García de Jalón, J., Bayo, E.: Kinematic and Dynamic Simulation of Multibody Systems. Springer Verlag (1993) 
22. Kurita, K., Inoue, F., Furuya, N., Shiokawa, T., Natori, M.: Development of adaptive roof structure by variable geometry truss. In: International Symposium on Automation and Robotics in Construction, pp. 1-6 (2001)

23. LaValle, S.M.: Planning Algorithms. Cambridge University Press, New York (2006)

24. Lavalle, S.M.: Motion planning. Part I: The essentials. IEEE Robotics and Automation Magazine 18(1), 79-89 (2011)

25. Lavor, C., Liberti, L., Maculan, N.: The discretizable molecular distance geometry problem. Tech. rep., arXiv (2006)

26. Liberti, L., Lavor, C.: On a relationship between graph realizability and distance matrix completion. In: A. Migdalas (ed.) Optimization Theory, Decision Making, and Operations Research Applications, vol. 31, pp. 39-48. Springer (2013)

27. Manocha, D., Canny, J.: Efficient inverse kinematics for general 6R manipulators. IEEE Transactions on Robotics and Automation 10, 648-657 (1994)

28. Merlet, J.P.: Parallel Robots. Springer (2000)

29. Miura, K.: Variable geometry truss concept. Tech. Rep. 614, The Institute of Space and Astronautical Science (1984)

30. Miura, K., Furuya, H., Suzuki, K.: Variable geometry truss and its application to deployable truss and space crane arm. Acta Astronautica 12(7), 599-607 (1985)

31. Porta, J.M.: CuikSLAM: A kinematics-based approach to SLAM. In: IEEE International Conference on Robotics and Automation, pp. 2436-2442 (2005)

32. Porta, J.M., Ros, L., Thomas, F.: Inverse kinematics by distance matrix completion. In: International Workshop on Computational Kinematics (2005)

33. Porta, J.M., Ros, L., Thomas, F.: On the trilaterable six-degree-of-freedom parallel and serial manipulators. In: IEEE International Conference on Robotics and Automation, pp. 960-967 (2005)

34. Porta, J.M., Ros, L., Thomas, F., Corcho, F., Cantó, J., Pérez, J.J.: Complete maps of molecular-loop conformational spaces. Journal of Computational Chemistry 28(13), 2170-2189 (2007)

35. Porta, J.M., Ros, L., Thomas, F., Torras, C.: Solving multi-loop linkages by iterating 2 D clippings. In: F. Thomas, J. Lenarcic (eds.) Advances in Robot Kinematics, pp. 255-264. Kluwer Academic Publishers (2002)

36. Porta, J.M., Ros, L., Thomas, F., Torras, C.: A branch-and-prune algorithm for solving systems of distance constraints. In: IEEE International Conference on Robotics and Automation, pp. 342-348 (2003)

37. Porta, J.M., Ros, L., Thomas, F., Torras, C.: A branch-and-prune solver for distance constraints. IEEE Transactions on Robotics 21(2), 176-187 (2005)

38. Porta, J.M., Thomas, F.: Closed-form position analysis of variable geometry trusses. Submitted

39. Porta, J.M., Thomas, F.: Sensor localization from distance and orientation constraints. Submitted

40. Raghavan, M.: The Stewart platform of general geometry has 40 configurations. ASME Journal of Mechanical Design 115, 277-282 (1993)

41. Rikun, A.D.: A convex envelope formula for multilinear functions. Journal of Global Optimization 10, 425-437 (1997)

42. Rodríguez, A., Basañez, L., Celaya, E.: A relational positioning methodology for robot task specification and execution. IEEE Transactions on Robotics 24(3), 600-611 (2008)

43. Rojas, N.: Distance-based formulations for the position analysis of kinematic chains. Ph.D. thesis, Institut de Robòtica i Informàtica Industrial (2012)

44. Rojas, N., Borràs, J., Thomas, F.: The octahedral manipulator revisited. In: IEEE International Conference on Robotics and Automation, pp. 2293-2298 (2012)

45. Rojas, N., Thomas, F.: Application of distance geometry to tracing coupler curves of pin-jointed linkages. ASME Journal of Mechanisms and Robotics 5(2), 021,001 (2013)

46. Rojas, N., Thomas, F.: The closure condition of the double banana and its application to robot position analysis. In: IEEE International Conference on Robotics and Automation, pp. 4641-4646 (2013) 
47. Rojas, N., Thomas, F.: The univariate closure conditions of all fully-parallel planar robots derived from a single polynomial. IEEE Transactions on Robotics 29(3), 758765 (2013)

48. Rosales, C., Porta, J.M., Ros, L.: Grasp optimization under specific contact constraints. IEEE Transactions on Robotics 29(3), 746-757 (2013)

49. Rosales, C., Ros, L., Porta, J.M., Suárez, R.: Synthesizing grasp configurations with specified contact regions. International Journal of Robotics Research 30(4), 431-443 (2011)

50. Rull, A., Porta, J.M., Thomas, F.: Distance bound smoothing under orientation constraints. In: IEEE International Conference on Robotics and Automation, pp. 1431$1436(2014)$

51. Siméon, T., Laumond, J.P., Cortés, J., Sahbani, A.: Manipulation planning with probabilistic roadmaps. International Journal of Robotics Research 23(7-8), 729-746 (2004)

52. Stoughton, R.S., Tucker, J.C.: A variable geometry truss manipulator for positioning large payloads. In: American Nuclear Society meeting on robotics and remote systems (1995)

53. Su, Y., Duan, B., Nan, R., Peng, B.: Mechatronics design of stiffness enhancement of the feed supporting system for the square-kilometer array. IEEE/ASME Tranactions on Mechatronics 8(4), 425-430 (2003)

54. Sultan, C., Corless, M., Skelton, R.E.: Tensegrity flight simulator. Journal of Guidance, Control, and Dynamics 26(6), 1055-1064 (2000)

55. Thomas, F.: An approach to the movers' problem that combines oriented matroid theory and algebraic geometry. In: IEEE International Conference on Robotics and Automation, vol. 3, pp. 2285-2293 (1995)

56. Thomas, F.: Solving geometric constraints by iterative projections and backprojections. In: International Conference on Robotics and Automation, pp. 1789-1795 (2004)

57. Thomas, F.: Computing cusps of $3 \mathrm{R}$ robots using distance geometry. In: International Symposium on Advances in Robot Kinematics (2014)

58. Ting, Y., Yu-Shin, Y.C., Jar, H.C.: Modeling and control for a Gough-Stewart platform CNC machine. International Journal of Robotics Systems 21(11), 609-623 (2004)

59. Trinkle, J.C., Milgram, R.J.: Motion planning for planar n-bar mechanisms with revolute joints. In: IEEE/RSJ International Conference on Intelligent Robots and Systems, vol. 3, pp. 1602-1608 (2001)

60. Wedemeyer, W.J., Scheraga, H.: Exact analytical loop closure in proteins using polynomial equations. Journal of Computational Chemistry 20(8), 819-844 (1999)

61. Wohlhart, K.: Position analyses of open normal Assur groups A(3.6). In: ASME/IFToMM International Conference on Reconfigurable Mechanisms and Robots, pp. 88-94 (2009) 